\title{
A Design Methodology for the Prediction of Squeeze Film Stiffness and Damping Characteristics in Hybrid Journal Bearings under Pulsatile Load Conditions.
}

\author{
D. Kinnear, Dr R.Mishra and Professor W. Weston \\ Department of Engineering and Technology, School of Computing and Engineering, University of Huddersfield, \\ Queensgate, Huddersfield, UK \\ C. Padgett, Padgett Racing Motorcycles, Batley, W Yorkshire, UK
}

\begin{abstract}
A theoretical investigation into the prediction and measurement of squeeze film stiffness and damping for double hole entry hybrid journal bearings has been undertaken together with a review of previous work to identify critical bearing parameters. The analysis used a partial differentiation of the Poiseuille and Hagen-Poiseuille flow equations to develop a mathematical model for the bearing squeeze film. A novel transfer function between bearing displacement and load enabled bearing stiffness and damping coefficients to be obtained from the subsequent frequency response characteristics.

Parameter maps were developed from the model for both stiffness and damping and show that the squeeze film stiffness is a function of bearing clearance and squeeze film pressure and is independent of fluid viscosity with damping being a function of viscosity and clearance.
\end{abstract}

\section{Key words}

Hybrid bearings, Squeeze film effect, IC Engine bearings, Power saving, Friction losses.

\section{Introduction}

Journal bearings comprise a plain shell that enables a shaft to rotate within the bearing housing, a form of lubrication is added to improve the characteristics of the bearing. Further development saw the shaft supported on a film of oil that provides opportunity for high accuracy, high loading and precision application. Operating on a film of oil is of great interest to engineers as it maintains a gap between the bearing surfaces that considerably reduces wear and improves bearing characteristics. In IC engines, approximately one third of engine power is lost to friction, with $15 \%$ of mechanical losses occurring in the crankshaft bearings so bearing design assumes considerable importance.

Engine bearings are usually of the journal type because they can be designed to be non contacting and to have an improved stiffness over rotating element bearings, an important factor when the impulsive nature of the load is considered. Also the use of rolling element bearings in crankshafts normally necessitates a built up crankshaft an unnecessary design complication.

There are two main effects that can be observed within journal bearings, these are the hydrostatic and hydrodynamic effects. The hydrostatic effect, Figure 1.1, arises from balancing the force exerted by the load with the pressure of the lubricant within the bearing, the balance of these two forces is known as the hydrostatic effect and the lubricant is supplied under pressure to support the applied load on the bearing. This effect is most suitable for low speed applications where the oil is less turbulent and the pressure required to support the applied load is predictable. Bearings designed with this effect provide excellent smoothness provided from startup, but are limited by the speed to maintain this effect.

The hydrodynamic effect, Figure 1.2, is observed in highspeed applications. The hydrodynamic pressures that separate the two bearing surfaces are generated as a consequence of the shaft rotating within the bearing housing. The moving elements of the bearing drag the lubricant by means of viscous forces into the converging gap between the two surfaces. This prevents the surfaces of the bearing contacting each other. The main draw back of bearings operating on this effect alone is the start-up with the speed not being enough to provide this effect initially.

Combining these effects enables the possibility to operate a no wear bearing at both low and high speeds. Bearings operating with both hydrostatic and hydrodynamic are known as hybrid bearings. They are normally designed using steady state load criteria with load disturbances being accommodated by the 'squeeze film' effect that is described as the stiffness and damping generated by the 
oil film being compressed and 'squeezed' out through the bearing lands The squeeze film gives the bearings high damping capacity and shock resistance whilst the hydrostatic and hydrodynamic effects enable the pressure of the lubricant to be maintained.

Journal bearings find applications in internal combustion engines e.g. crankshaft and connecting rods but, to date, there is no evidence of systematic hybrid bearing design being employed. However, a classical hybrid design has been undertaken in the Department of Engineering and Technology, University of Huddersfield for a high speed two stroke $125 \mathrm{cc}$ motorcross engine crankshaft bearing, Figure 2, which has resulted in implausibly high design supply pressures. The conclusion is that the squeeze film effect enables conventional journal bearings to operate successfully under pulsatile load conditions and cannot be ignored in hybrid bearing design.

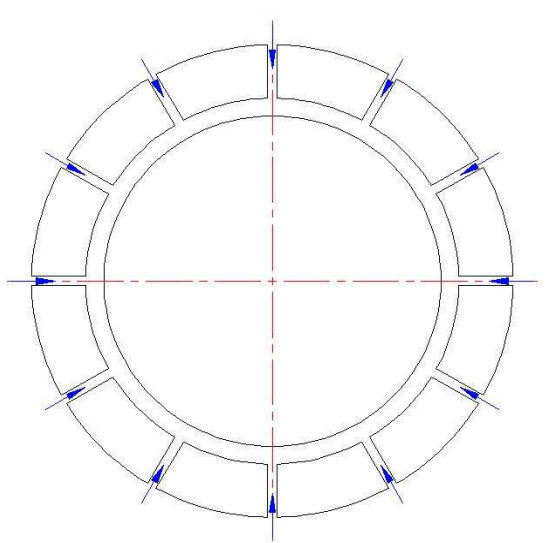

Figure 1.1 - Hydrostatic Effect

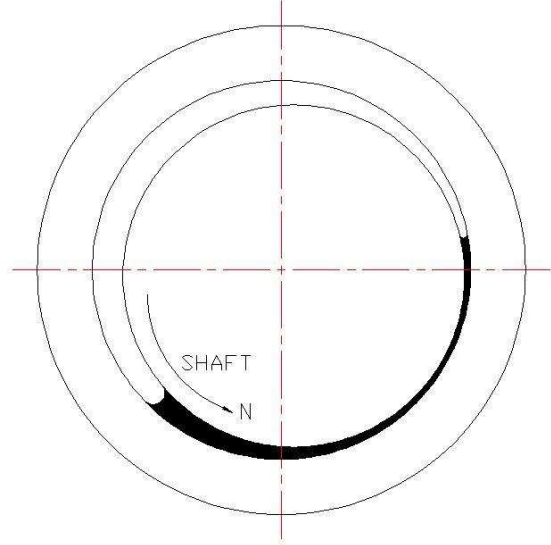

Figure 1.2 - Hydrodynamic Effect

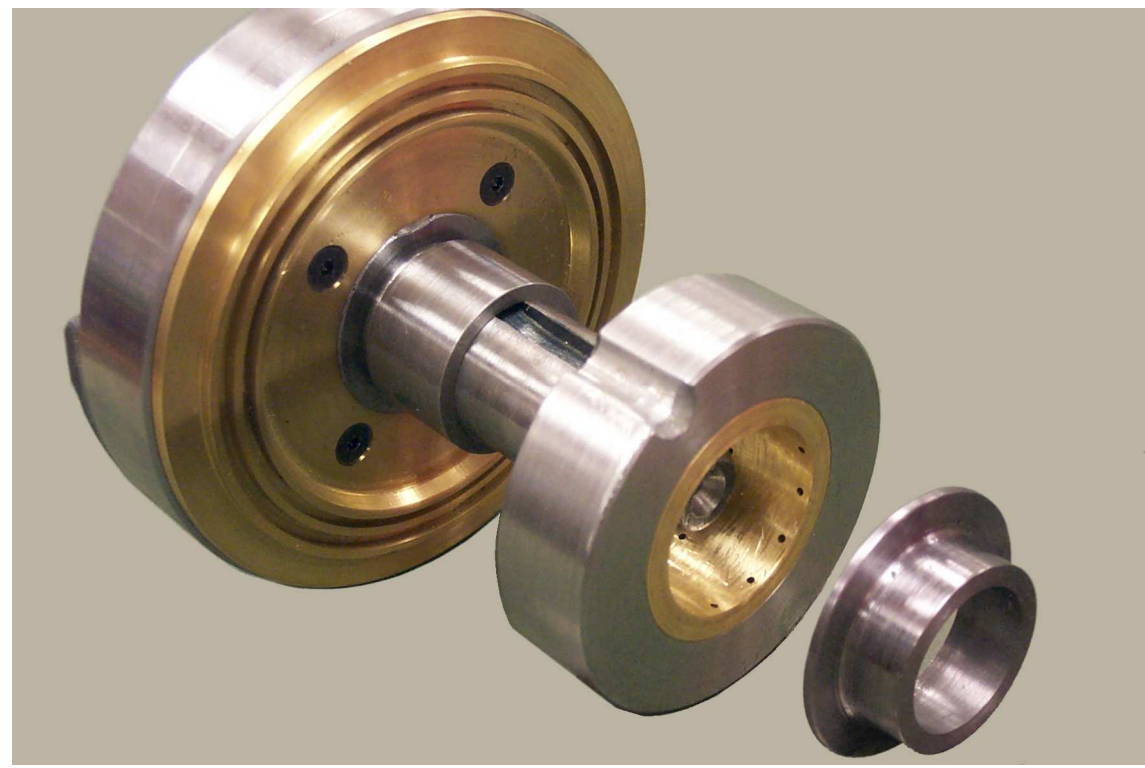

Figure 2 - Engine Crankshaft Hole Entry Hybrid bearing

This paper explores the possibility of hybrid bearings using the squeeze film effect being used for pulsatile load applications typically found in internal combustions engines. The theory develops a transfer function that yields bearing stiffness and damping and parameter maps for stiffness and damping.

\section{Review of Previous Work}

Hybrid bearings have been researched for many different reasons in many configurations and aim to combine the benefits of hydrostatic bearings and hydrodynamic bearings.
The hydrodynamic stiffness, hydrostatic stiffness and squeeze film were derived, by W.B. Rowe, W. Weston and D. Koshal [1], for any number of feed inlets. The research covered recessed bearings and plain slot entry bearings. Koshal and Rowe [2,3] also investigated various arrangements of hole entry hybrid bearings and were able to demonstrate that having two rows of inlet lubrication sources situated near the edges of the bearing provided greater load carrying capacity.

Many researchers have discussed the design of hybrid bearings but Rowe et al [4] investigated the design with particular reference to hole-entry configurations for different configurations of journal bearings. Ease of 
manufacture and the performance of hybrid bearings were emphasised.

Hole-entry hybrid bearing systems have been investigated like many journal bearings in respects to the lubrication, thermal effects and rotational effects. S.C. Sharma et al [6] produced a theoretical model to study the performance of bearings with respect to temperature change of the lubrication affecting the viscosity. The research concluded that the variation of viscosity due to temperature rise of the lubricant had quite a substantial influence on the static and dynamic performance of the hybrid bearing system. Designers of hybrid bearing systems must consider the effects carefully with the design in order to obtain the desired performance characteristics.

$\mathrm{Su}$ and Lie [7] investigated the rotational effects of hybrid bearings with various feeding mechanisms, including orifice, capillary and porous mechanisms. Investigating the multi-array of feeding methods determined that load capacity was increased with more supply ports at low speeds and fewer at high speeds. This highlights the importance of bearing design for the desired application and the difficulties in designing hybrid bearings.

Kumar et al [8] however further studied the design and hole layout of hole-entry hybrid journal bearings using a finite element method and investigated different configurations for both single hole and paired holes. The paper concluded the single hole with locally concentrated holes to be more suitable for high speed and high load support, in contrast to the work by D. Koshal and W.B. Rowe [2,3] who had determined two rows of holes located near the edges of the bearing provided an enhanced performance.

Internal combustion engines have recently been investigated by L. Morais and L.A. Ferreira [9] who investigated optimising a journal bearing for mass balancing systems. They investigated design parameters using the finite difference method and were able to optimise dimensions accordingly with the load application.

\section{Mathematical Model}

The method uses the Poiseuille and Hagen Poiseuille equations to determine the pressure induced viscous flow from the bearing lands and through the supply capillaries.

Referring to Figure 3, the Poiseuille equation applied to the bearing lands gives:

$Q_{A}=2 Z H^{3}\left(P_{s q}-P_{\text {laby }}\right) /(12 \eta L / 2)$

$Q_{C}=2 L H^{3}\left(P_{s q}-\mathrm{P}_{\text {laby }}\right) /(12 \eta \mathrm{Z} / 2)$

where $\mathrm{Z}=$ width[m], $\mathrm{H}=\mathrm{Gap}[\mathrm{m}], \mathrm{L}=$ Length[m], $\mathrm{Q}_{\mathrm{A}}=$ Axial land Flow and $\mathrm{Q}_{\mathrm{C}}=$ Circumferential land Flow
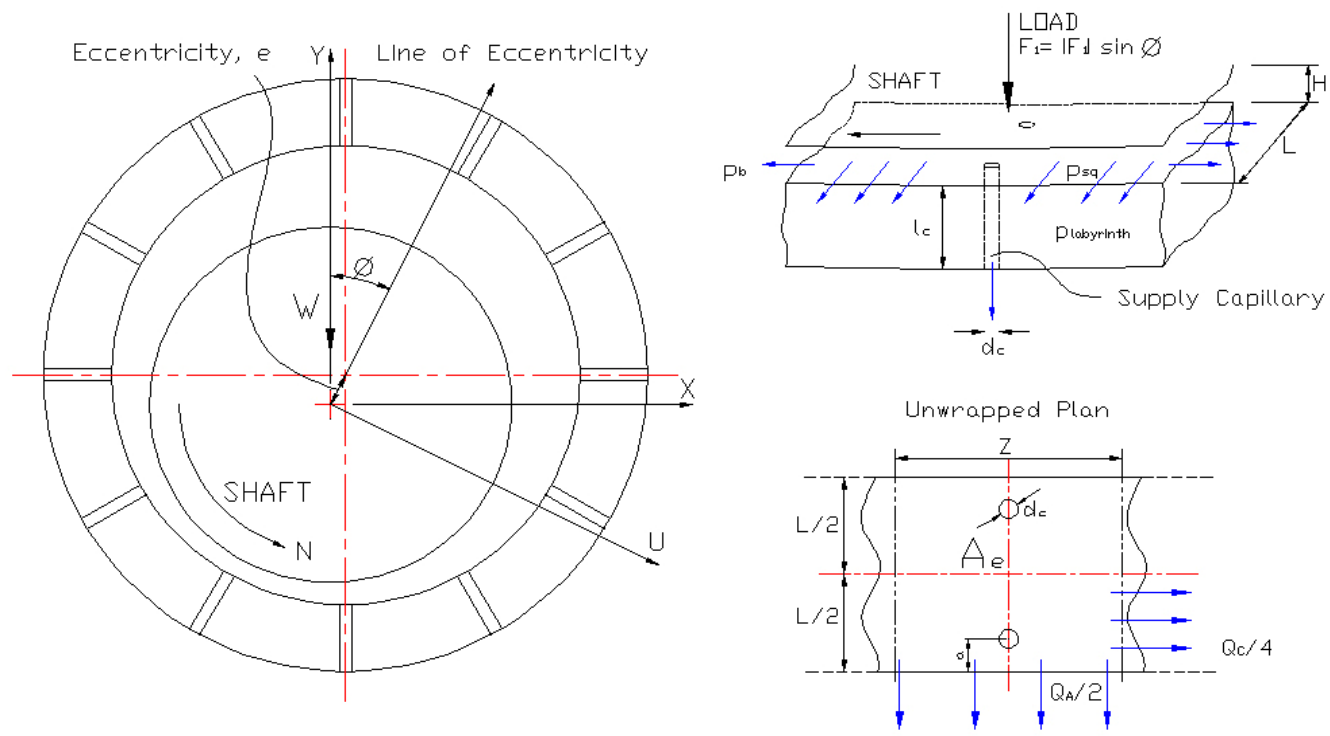

Figure 3 - Duel Hole Entry Hybrid Bearing

Partial differentiation with respect to the two variables, gap $\mathrm{h}$ and squeeze pressure $p_{s q}$ will yield the small perturbations in axial and circumferential low to give:

$q_{s q}=q_{A}+q_{C}$ 
where

$q_{A}=\left(\frac{\partial Q_{A}}{\partial P_{s q}}\right) p_{s q}+\left(\frac{\partial Q_{A}}{\partial H}\right) h$

$q_{C}=\left(\frac{\partial Q_{C}}{\partial P_{s q}}\right) p_{s q}+\left(\frac{\partial Q_{C}}{\partial H}\right) h$

$\frac{\partial Q_{A}}{\partial P_{s q}}=\frac{Z H^{3}}{12 \eta L / 2} \times 2$

$\frac{\partial Q_{A}}{\partial H}=\frac{3 Z H^{3} P_{s q}}{12 \eta L / 2} \times 2$

$\frac{\partial Q_{C}}{\partial P_{s q}}=\frac{L H^{3}}{12 \eta Z / 2} \times 2$

$\frac{\partial Q_{C}}{\partial H}=\frac{3 L H^{3} P_{s q}}{12 \eta Z / 2} \times 2$

Applying the Hagen Poiseuille equation to the feed capillaries yields:

$$
q_{O R}=\frac{\left(P_{b r g}-P_{s q}\right) \times 2 N}{K_{C} \eta}
$$

where $\mathrm{N}=$ the number of orifice pairs in the bearing and $K_{C}$ is the capillary factor $=\frac{128 l_{C}}{\pi d_{C}{ }^{4}}$

However, a typical bearing gap is in the order of $25 \mu \mathrm{m}$, compared to a typical oil way and orifice diameter of approximately $2.5 \mathrm{~mm}$. Thus most of the squeeze flow gets forced back up the supply capillaries, making for a bearing with a low stiffness. For this reason, a fast acting non return valve located in the supply line has been designed. This uses a cantilevered 'flapper' with a natural frequency in the order of $7500 \mathrm{~Hz}$, adequate to give a flat frequency response for most engine applications. The displaced volume flow is defined as:

$$
q_{\text {vol }}=A_{e} H \text { where } A_{e}=L \times Z
$$

and compressibility flow $q_{C}$ as:

$q_{C}=\frac{V}{K} P_{s q}$ where $V=L Z H$ and

\section{$K=$ Bulk Modulus}

Summing the flow equations yields:

$$
q_{O R}=q_{s q}+q_{\text {vol }}+q_{C}
$$

and substituting into (11) yields:

$0=\left\{\frac{Z H^{3}}{3 \eta L}+\frac{L H^{3}}{3 \eta Z}\right\} p_{s q}+\left\{\frac{Z H^{2}}{\eta L}+\frac{L H^{2}}{\eta Z}\right\} P_{s q} h+A_{e}+\frac{V}{K} P_{s q}$

Write $p_{s q}=\frac{w}{A_{e}}, h=-y$,
$B=\left\{\frac{Z H^{3}}{3 \eta L}+\frac{L H^{3}}{3 \eta Z}\right\}$

and $C=\left\{\frac{Z H^{2}}{\eta L} \frac{3 L H^{2}}{\eta Z}\right\} P_{s q}$

to obtain the relationship between bearing displacement $\mathrm{y}$ and load $\mathrm{w}$

$$
y\left(C+A_{e} D\right)=\frac{w}{A_{e}}\left(B+\frac{V}{K} D\right)
$$

This yields the transfer function:

$$
\frac{y}{w}=K_{o} \frac{\left(1+\tau_{N} D\right)}{1+\tau_{D} D}
$$

where

$$
\begin{aligned}
& K_{o}=B / A_{e} C, \\
& \tau_{N}=V / K B \\
& \tau_{D}=A_{e} / C
\end{aligned}
$$

This represents the generalised mathematical model for hole entry hybrid bearings under pulsatile load, as in internal combustion applications. The transfer function has not been 'normalised' since the researchers wish to retain an understanding of the variables involved. This is important as is the development of the fast response nonreturn valve in order to maintain the desired bearing stiffness. The gain and phase characteristics shown in Figure 4 are obtained by writing $D=j \omega$ in the normal way and will yield the bearing stiffness, $\mathrm{k}=\frac{1}{K_{o}}$, and damping $c=\frac{\tau_{D}}{K_{o}}$

\section{Parameter Mapping for Stiffness, $k[\mathrm{~N} / \mathrm{m}]$ and Damping, $\mathrm{c}\left[\mathrm{N} / \mathrm{ms}^{-1}\right]$-Figures 5\&6}

Parameter maps for stiffness and damping (Figures 5\&6)) are obtained from the mathematical model and are shown below using the data from the bearing under investigation, where:

Bearing Width, $\mathrm{Z}=25 \mathrm{~mm}$,

Bearing Length, $\mathrm{L}=15 \mathrm{~mm}$,

Bearing gap, $\mathrm{H}=25 \mu \mathrm{m}$ and

Dynamic Viscosity $=5 \times 10^{-2} \mathrm{Ns} / \mathrm{m}^{2}$

Bearing stiffness, $k=7.95 \times 10^{-4} P_{s q} / H$

(from $K=K_{0}^{-1}=A_{e} C / B$ ) and Bearing damping,

$c=0.186 \times 10^{-6} \eta / H^{3}\left\lfloor N / m^{-1}\right\rfloor$ 

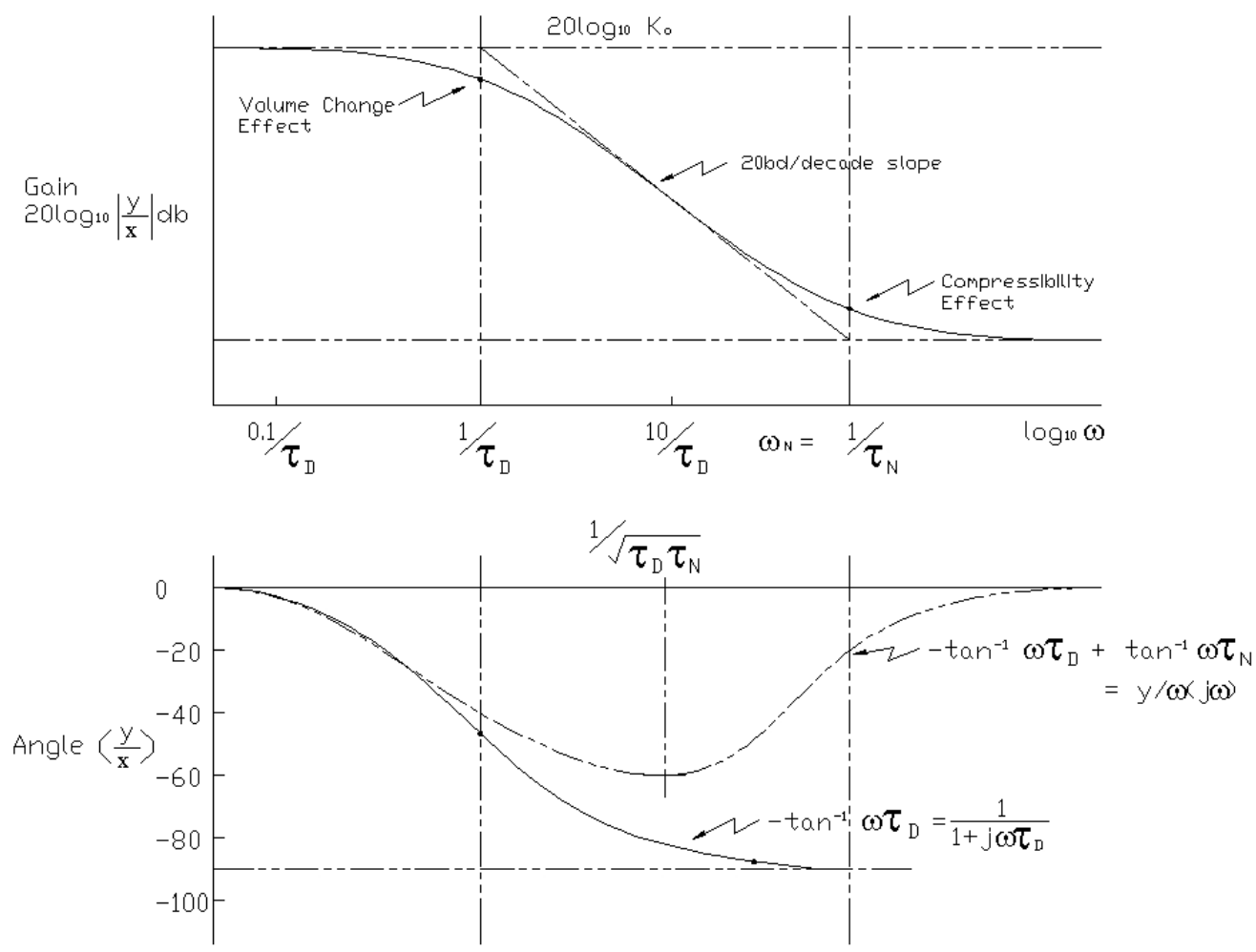

Figure 4 - Squeeze Film Frequency Response Characteristics

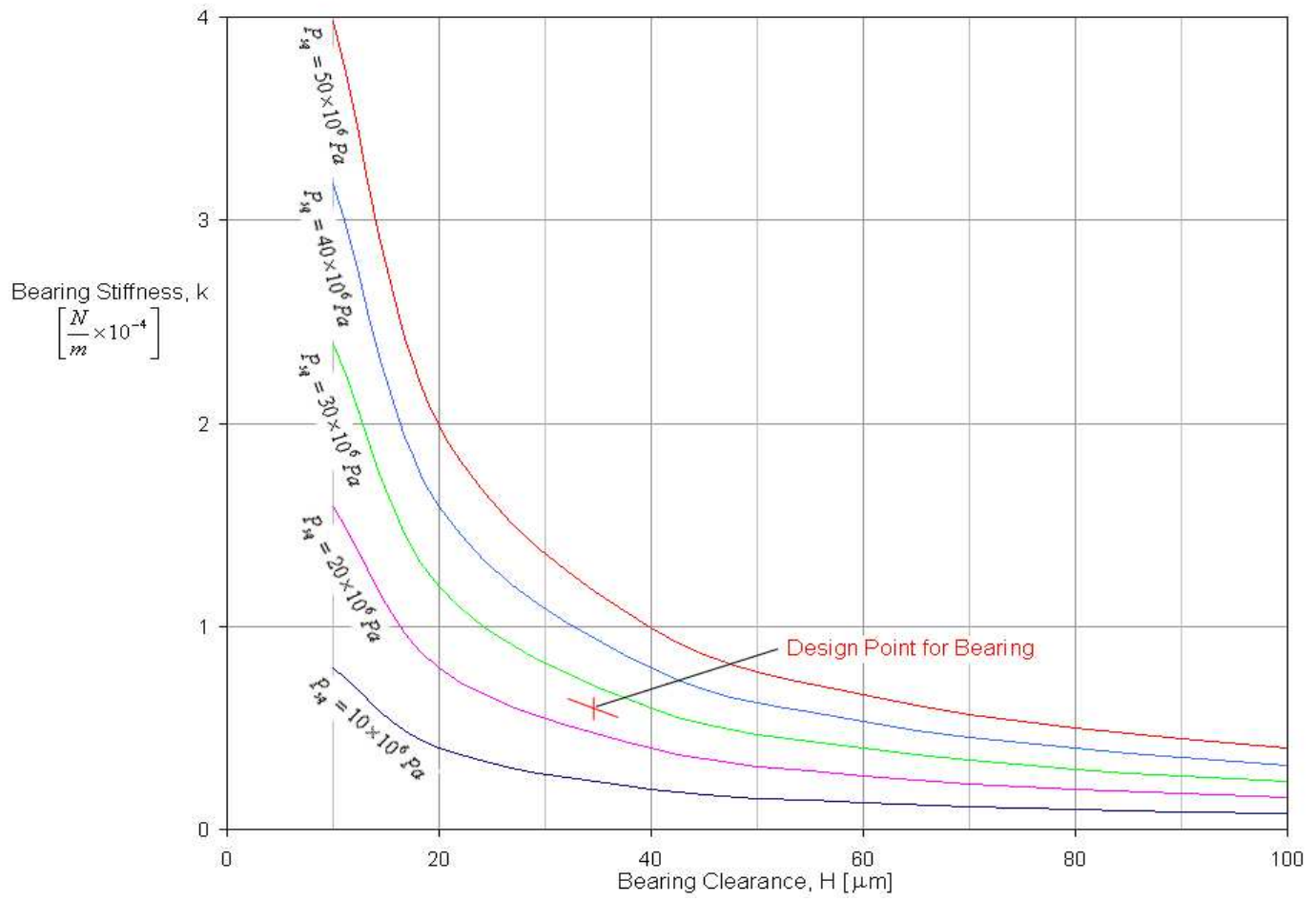

Figure 5 - Stiffness vs Clearance 


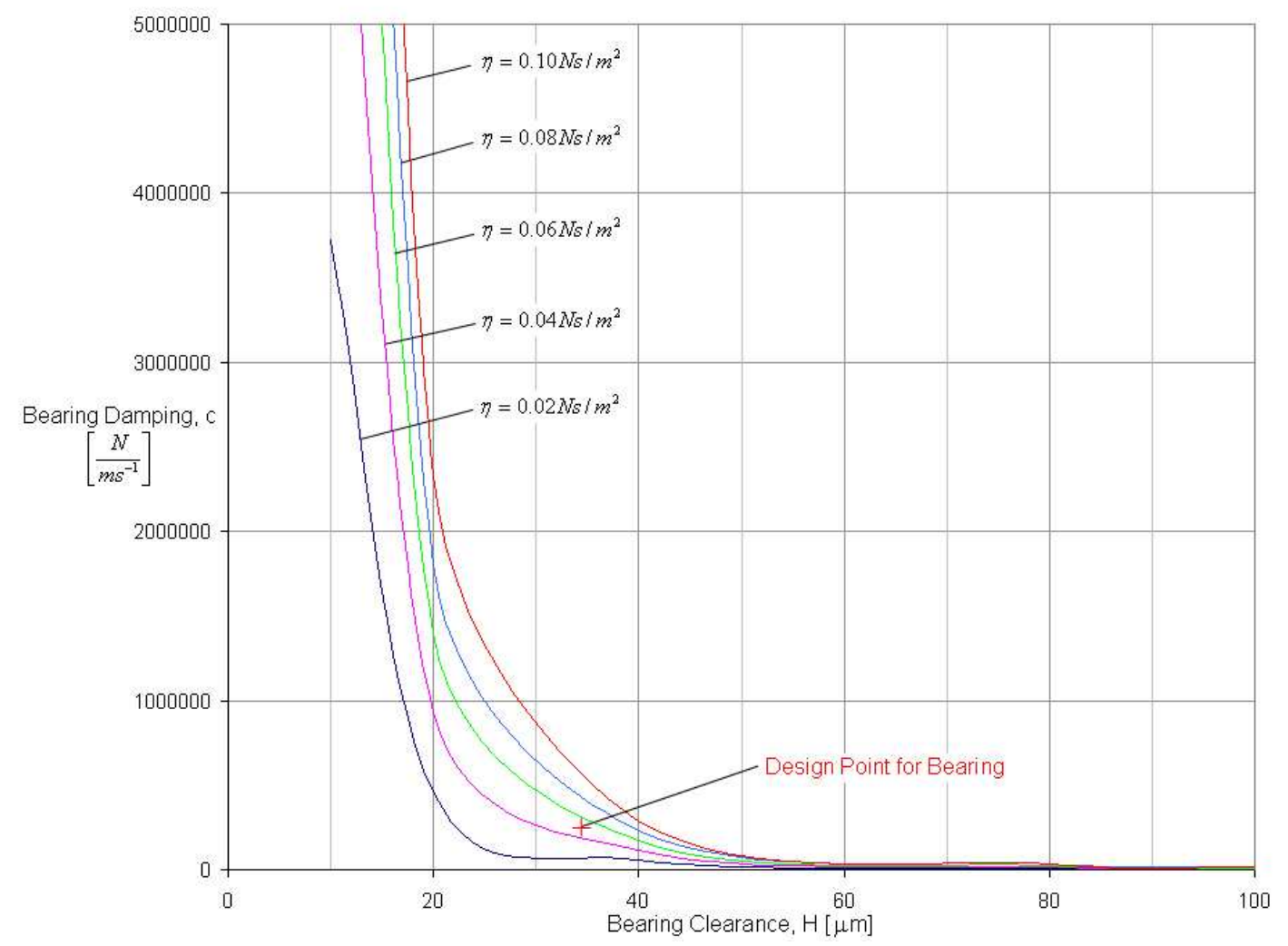

Figure 6- Damping vs Clearance

\section{A Critique of the Design Methodology}

Journal bearing design is well established with petrochemical companies often providing design criteria based on performance parameters such as eccentricity ratio vs load capacity. The 1969 BP publication: Lubrication Theory and its Application (10) is an example of fundamental journal bearing design. Design methodology was significantly improved by WB Rowe (1-5) and developed the concept of hybrid hydrostatic/hydrodynamic operation which defined the bearing size for a given load, dependant on the power ratio $\mathrm{K}$, the ratio between frictional power and pumping power. Both design methodologies have been extensively used with Rowe's proposals significantly improving bearing performance. Bearing design which enhances performance and lowers frictional losses is a very necessary criterion in modern engine design which sees one third of engine power lost by friction with approximately $15 \%$ of mechanical losses occurring in the crankshaft bearings. However, neither technique addresses the crankshaft bearing load configuration which occurs in internal combustion engine applications with unsteady loads, almost impulsive loads of typically two tonnes occurring each engine cycle. Bearing deflections under this type of loading incur large strain energy losses necessitating the development of bearings with good stiffness characteristics which enable these losses to be minimised. The method described in this paper enables the effect of impulsive type internal combustion engine crankshaft loads to be considered in the bearing design process and is based on a method described by Rowe(5) and developed at the University of Huddersfield. It enables the design to be systematically addressed to reduce bearing clearance and improve stiffness which will improve the power quality of the engine.

\section{Discussion}

The technique used has the advantage of being both simple and effective and enables a good understanding of the physical parameters and phenomena involved. It has been assumed that the flow is laminar, a reasonable assumption given the small clearances used and that Couette flow, which yields the flow due to shaft rotation, is of a second order effect.

The model provides a transfer function for shaft displacement against bearing load in the frequency domain and assumes that the pulsatile load application found in internal combustion engines is harmonic. The transfer function has two break frequencies, the lower of which yields the damping factor, the higher being due to fluid compressibility.

The system gain as excitation frequency approaches zero yields the bearing stiffness with the high stiffness values being obtained through the use of a specially designed non-return valve in the supply line, which prevents oil being squeezed back by the large squeeze film pressures developed. This is in preference to the use of restrictors 
in the bearing orifices, the normal method, which both restricts flow and the ability of the bearing to remove heat energy.

A parameter map for the gain shows the stiffness to be proportional to the bearing squeeze film pressure and inversely proportional to clearance, whereas the parameter map for damping showed damping to be proportional to viscosity and inversely proportional to clearance cubed.

The design methodology resulting from the mathematical model needs to be informed experimentally for full confidence in the technique. It would be an interesting addition to compare the results from this direct method with a computational fluid dynamics model providing more information to link in with the mathematical model and theoretical results.

\section{References}

[1] Rowe, W.B., Weston, W, Koshal, D, "Static and Dynamic Properties of Concentric Hydrostatic Journal Bearings by Small Displacement Analysis", Euromech Conference on Hydrodynamic Lubrication of Bearings, Fiat, Turin, Oct. 1979

[2] Koshal, D, Rowe, W.B., "Fluid-Film Journal Bearings Operating in a Hybrid Mode: Part I - Theoretical Analysis and Design”, Journal of Lubrication Technology, ASME, Vol. 103, pp. 558-65, Oct. 1981
3] Koshal, D, Rowe, .B., "Fluid-Film Journal Bearings Operating in a Hybrid Mode: Part II - Experimental Investigation", Journal of Lubrication Technology, ASME, Vol.

[4] Rowe, W.B., Xu, S.X., Chone, F.S., Weston, W., "Hybrid journal bearings with particular reference to hole-entry configurations", Tribology International, Vol. 15, pp. 339-48. Dec. 1982

[5] Rowe, W.B., Hydrostatic and Hybrid Bearing Design, Butterworths, 1983

[6] Sharma, S.C., Kumar, V, Jain, S.C., Nagaraju, T, "Study of hole-entry hybrid journal bearing system considering combined influence of thermal and elastic effects", Tribology International, Vol. 39, pp. 715-25, Jul 2006

[7] Su, J.C.T., Lie, K.N., "Rotational effects on hybrid hydrostatic/hydrodynamic journal bearings", Industrial Lubrication and Tribology, Vol. 53, pp. 261-69, Dec. 2001

[8] Kumar, V, Aggarwal, J, Ghai, S.K., "Performance Characteristics of Hole-entry Hybrid Journal Bearings of Different Configurations", Journal of Institution of Engineer (India), Vol. 85, pp. 20-25. Apr. 2004

[9] L, Morais, L.A., Ferreira, "Journal bearings optimisation for mass balancing systems used in internal combustion engines", Industrial Lubrication and Tribology, Vol. 58, pp. 295-302, 2006

[10] Anon Lubrication Theory and its Application, London, BP trading Ltd, 196 